\title{
Can Pr-Doped YAP Scintillator Perform Better?
}

\author{
Martin Nikl, Jiri A. Mares, Anna Vedda, Mauro Fasoli, Valentin Laguta, Eva Mihokova, Jan Pejchal, \\ Mariya Zhuravleva, Akira Yoshikawa, and Karel Nejezchleb
}

\begin{abstract}
A numerous set of Pr-doped YAP single crystals was prepared using Czochralski and micro-pulling down methods. Crystals codoped by bi-, tri- and tetravalent ions were prepared by the latter method as well. The scintillation performance of all the prepared materials was evaluated using radioluminescence, photoelectron yield and scintillation decay measurements at room temperature. The influence of shallow traps was studied by means of thermoluminescence in the 10-350 $\mathrm{K}$ temperature range. Though the intrinsic scintillation efficiency of Pr-doped YAP is higher than that of YAP:Ce, enhanced delayed radiative recombination processes are found to be responsible for lower-than-expected photoelectron yield of the former system. The possibilities of further optimization of Pr-doped YAP scintillator are discussed.
\end{abstract}

Index Terms-Luminescence, photoelectron yield, Pr-doped YAP, scintillator, thermoluminescence.

\section{INTRODUCTION}

$\mathbf{T}$ $\mathrm{HE}$ luminescence characteristics of $\mathrm{Ce}^{3+}$-doped $\mathrm{YAlO}_{3}$ (YAP) single crystal, namely an intense emission band at about $360 \mathrm{~nm}$ and a decay time of the $5 \mathrm{~d}_{1}-4 \mathrm{f}$ transition of $\mathrm{Ce}^{3+}$ of about $18 \mathrm{~ns}$, were reported in the early 1970 s by M. Weber [1]. The favorable properties of this material as fast scintillator were revealed some years later [2]. A considerable effort to increase its low density and low effective atomic number $\left(\mathrm{Z}_{\mathrm{eff}}\right)$ by partial or complete substitution of Lu for Y ion was made in the 1990s (see [3] for a review). However, the growth of such crystals was

Manuscript received June 29, 2009; revised September 25, 2009; accepted February 04, 2010. Date of current version June 16, 2010. This work was supported in part by the Czech GAAV project M100100910, in part by DESY project I-20090104 EC, and in part by the Italian CARIPLO Foundation project "Energy transfer and trapping phenomena in nano-structured scintillator materials" (2008-2011). JSPS Postdoctoral Fellowship for Foreign Researchers Program P08055 (JP) is also acknowledged.

M. Nik1, J. A. Mares, and V. Laguta are with the Institute of Physics AS CR v. v. i., 16253 Prague, Czech Republic (e-mail: nik1@fzu.cz; amares@fzu.cz; laguta@fzu.cz).

A. Vedda and M. Fasoli are with the Department of Materials Science, University of Milano-Bicocca, 20125 Milan, Italy (e-mail: anna.vedda@mater. unimib.it; mauro.fasoli@mater.unimib.it).

E. Mihokova is with the Institute of Physics AS CR v. v. i., 16253 Prague, Czech Republic , and also with the Department of Materials Science, University of Milano-Bicocca, 20125 Milan, Italy (e-mail: mihokova@fzu.cz).

J. Pejchal is with the Institute of Physics AS CR v. v. i., 16253 Prague, Czech Republic, and also with the Institute of Multidisciplinary Research for Advanced Materials (IMRAM), Tohoku University, Sendai 980-8577, Japan (e-mail: pejchal@fzu.cz).

M. Zhuravleva and A. Yoshikawa are with the Institute of Multidisciplinary Research for Advanced Materials (IMRAM), Tohoku University, Sendai 9808577, Japan (e-mail: mzhuravl@utk.edu; yosikawa@tagen.tohoku.ac.jp).

K. Nejezchleb is with the CRYTUR, Ltd., 51119 Turnov, Czech Republic (e-mail: nejezchleb@crytur.cz).

Color versions of one or more of the figures in this paper are available online at http://ieeexplore.ieee.org.

Digital Object Identifier 10.1109/TNS.2010.2043116 troubled by the narrow region of stability of the perovskite phase due to the smaller radius of the Lu cation.

$\mathrm{Pr}^{3+}$ doping was studied in a number of fluoride and oxide compounds in the search for photon cascade emission [4], while it was considerably less investigated for scintillation purposes [5]. Only recently, more systematic comparative studies were performed in the group of aluminum garnets and orthosilicates [6] where LuAG:Pr appeared as a new promising scintillator [7]. Pr doping was also pursued in the YAP host resulting in the high energy shifted $5 \mathrm{~d}_{1}-4 \mathrm{f}$ emission peaking at about 245-250 nm with the scintillation decay time of about $13 \mathrm{~ns}$ [8]. Other works confirmed such a fast scintillation response of the Pr-doped YAP and LuAP single crystals, however, with respect to the Ce-doped analogues these materials provide much lower light yield [9]-[12]. Based on the fact that both $\mathrm{Ce}^{3+}$ and $\mathrm{Pr}^{3+}$ centers in YAP should act as the hole traps and that the scintillation mechanism is supposed to be analogous in both cases the reason for such a discrepancy in the light yield is difficult to understand. Therefore further optimization of Pr-doped aluminum perovskite remains an open problem.

In this contribution we compare scintillation efficiency (radioluminescence intensity), photoelectron yield and scintillation decay in an extended set of YAP:Pr crystals prepared by three different technological methods. The effect of various codoping ions is studied as well. Furthermore, the differences in Ce-doped and Pr-doped YAP single crystals in the energy migration and trapping mechanisms are evidenced using the thermoluminescence measurements. Electron spin resonance provides an additional insight into the nature of the involved defects and traps. Based on the achieved understanding, we discuss possibilities of further optimization of Pr-doped aluminum perovskite scintillators.

\section{SAMPLES AND EXPERIMENTAL TECHNIQUES}

The YAP samples used in this study are i) standard Ce-doped crystal (0.5 at\%) grown by Czochralski technique $(\mathrm{Cz})$ with $4 \mathrm{~N}$ purity starting materials from molybdenum (Mo) crucible in CRYTUR Ltd (Czech Republic) which is used as a reference sample; the crystal with lower doping level $(0.05 \%)$ grown by Czochralski technique with $4 \mathrm{~N}$ purity starting materials from iridium (Ir) crucible in IMRAM, Tohoku university (Japan); ii) altogether twenty three $\mathrm{Pr}$-doped $(0.005-5 \%)$ and $(\mathrm{Mg}, \mathrm{Si}, \mathrm{Zr}$, $\mathrm{Hf}, \mathrm{Ga}, \mathrm{Mo}, 0.005-0.5 \%)$ codoped crystals grown in IMRAM, Tohoku University (Japan) by the micro-pulling down method (mPD) with $4 \mathrm{~N}$ purity starting materials from iridium crucible, (iii) Pr-doped crystal (0.5 and $1.1 \%$ ), Cz-grown with $4 \mathrm{~N}$ purity starting materials from molybdenum crucible in CRYTUR Ltd. All concentrations are given in molar percent and refer to the melt. From now on the samples will be denoted by abbreviations indicating the dopant, concentration, growth method 


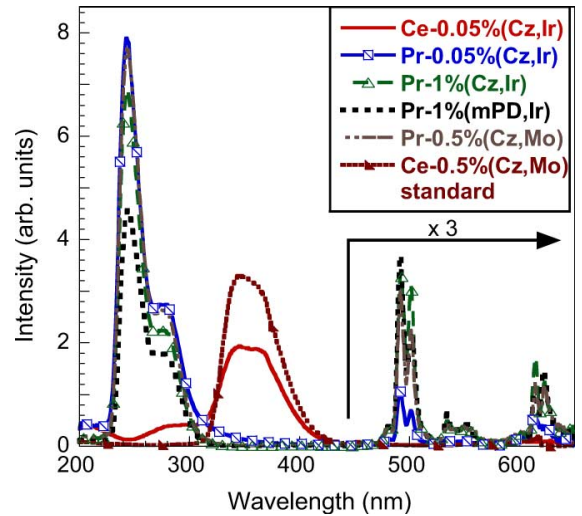

Fig. 1. Radioluminescence spectra of Ce and Pr-doped YAP at RT. Excitation by X-ray, $40 \mathrm{kV}$. Spectra are mutually comparable in an absolute scale.

and crucible type (for example, Pr-5\%(mPD,Ir)). The samples for experiments were prepared from parent crystals by cutting the plates of dimensions $7 \times 3 \times 1 \mathrm{~mm}^{3}(\mathrm{mPD})$ and $7 \times 7 \times$ $1 \mathrm{~mm}^{3}(\mathrm{Cz})$ and polishing them to an optical grade. Codoping of YAP:Pr crystals was used as a tool to change an internal equilibrium of the point defects in YAP host. In case of aliovalent codopants we follow the strategy successfully used by some of us in the case of trivalent-ion-doped $\mathrm{PbWO}_{4}$ [3], [13]). In case of trivalent codopants the goal was either to influence the concentration of antisite defects, the existence of which in YAP host was recently proved by NMR [14], or to use it for the band gap engineering. Recently we have successfully applied the latter concept in the Ga-admixed LuAG:Pr [15].

Radioluminescence spectra, excited by an X-ray tube (W cathode), were measured by a modified 199S Edinburgh Instrument spectrofluorometer equipped with single grating monochromators and a photon counting photomultiplier-based detector. Conditions of experiment, including geometry, were carefully kept the same to enable comparison of the spectra within the sample set in an absolute scale (Figs. 1 and 6 in Section III). Photoluminescence and scintillation decays were measured by the same set-up using the time-correlated single photon counting method. $\mathrm{H}_{2}$-filled nanosecond flashlamp and ${ }^{22} \mathrm{Na}$ radioisotope were used for the excitation, respectively. In the latter case we used the concept of Bollinger and Thomas [16] with the fast plastic scintillator in front of the "Start" photomultiplier. Absorption spectra were measured by a UV-VIS_NIR Shimadzu UV-3101PC spectrophotometer. All these measurements were performed at room temperature (RT).

At RT the $\mathrm{N}_{\text {phels }}$ photoelectron yield, energy resolution (calculated from FWHM of the photopeak) and proportionality of $\mathrm{N}_{\text {phels }}$ were measured by new set-up with a hybrid photomultiplier (HPMT), model PPO 475B, manufactured by DEP company, Roden, the Netherlands, and multichannel analyzer in the pulse-height-analysis mode [17], [18]. For smaller samples (mPD-grown) the $\mathrm{N}_{\text {phels }}$ measurements were carried out under the excitation of ${ }^{109} \mathrm{Cd}(22.2$ and $88 \mathrm{keV})$ and ${ }^{241} \mathrm{Am}(\sim 16$ and $59.6 \mathrm{keV}$ ) because in this range the YAP host has much higher linear attenuation coefficient than in the range above $200 \mathrm{keV}$. For larger YAP:Ce and YAP:Pr Cz-grown samples with higher $\mathrm{N}_{\text {phels }}$ yield the measurements were carried out in the wider energy range up to $1275 \mathrm{keV}$ using also ${ }^{57} \mathrm{Co}$, ${ }^{22} \mathrm{Na}$ and ${ }^{137} \mathrm{Cs}$ energy lines. All the samples were measured at $1 \mu$ s shaping time and several selected Ce- and Pr-doped ones were additionally studied within a wider shaping time range of 0.5-10 $\mu$ s to compare their $\mathrm{N}_{\text {phels }}$ time dependence.

TSL measurements after $\mathrm{x}$-irradiation at $10 \mathrm{~K}$ and with a heating rate of $0.1 \mathrm{~K} / \mathrm{s}$ were performed in the $10-310 \mathrm{~K}$ temperature range with a home-made apparatus featuring a CCD detector (Jobin-Yvon Spectrum One 3000) coupled to a monochromator (Jobin-Yvon Triax 180) with 300 and 100 grooves $/ \mathrm{mm}$ gratings operating in the $278-713 \mathrm{~nm}$ and 138-1447 $\mathrm{nm}$ ranges. In this case x-ray irradiations were performed by a Philips 2274 tube operating at $20 \mathrm{kV}$.

\section{RESULTS AND DisCUSSION}

RT radioluminescence spectra of selected YAP samples doped exclusively with $\mathrm{Pr}$ are compared in absolute scale with those of Ce- $0.5 \%(\mathrm{Cz}, \mathrm{Ir})$ standard and Ce- $0.05 \%(\mathrm{Cz}, \mathrm{Ir})$ samples in Fig. 1.

The wide double band with maxima at about $242 \mathrm{~nm}$ and $280 \mathrm{~nm}$ is due to $\mathrm{Pr}^{3+}$ radiative transition from its $5 \mathrm{~d}_{1}$ level. The former peak includes transitions to ${ }^{3} \mathrm{H}_{4}$ and ${ }^{3} \mathrm{H}_{5}$ levels, while the latter includes those to ${ }^{3} \mathrm{H}_{6}$ and ${ }^{3} \mathrm{~F}_{x}(x=2,3,4)$. A better spectral resolution of separate transitions in the spectrum is achieved at low temperature [11]. An integral of emission spectra in the region of fast $5 \mathrm{~d}-4 \mathrm{f}$ emission $(200-450 \mathrm{~nm})$ can be considered as the measure of the overall scintillation efficiency. The integral values are higher in all the Cz-grown Pr-doped YAP samples with respect to that of the $\mathrm{Ce}-0.5 \%(\mathrm{Cz}, \mathrm{Mo})$ standard sample, see Table I. It is worth noting considerably lower radioluminescence intensity in the case of mPD grown sample: such effect has been already noticed in the case of systematically studied Yb-doped YAP [19], [20] and could be assigned to possibly inferior crystal quality with respect to $\mathrm{Cz}$-grown crystals and radial dependence of the dopant segregation which is a frequent problem in $\mathrm{mPD}$-grown crystals [21].

It is interesting to note the change of the intensity ratio of the $5 \mathrm{~d}-4 \mathrm{f}$ emission peaking at $242 \mathrm{~nm}$ and $4 \mathrm{f}-4 \mathrm{f}$ emission lines around $490 \mathrm{~nm}$ (from ${ }^{3} \mathrm{P}_{0}$ level) and $620 \mathrm{~nm}$ (from ${ }^{1} \mathrm{D}_{2}$ level): the $4 \mathrm{f}-4 \mathrm{f}$ emission intensity becomes relatively more intense with increasing Pr concentration. This effect has been already noticed [12], [22] and ascribed to a possible cross-relaxation effect between nearby lying $\mathrm{Pr}^{3+}$ ions [12]. Such a process can be enabled by the fact that $5 \mathrm{~d}_{1}-4 \mathrm{f}$ emission is located at 242 $\mathrm{nm}$, the half wavelength of that related to $(4 \mathrm{f}-4 \mathrm{f})^{3} \mathrm{P}_{0}-{ }^{3} \mathrm{H}_{4}$ radiative transition at about $490 \mathrm{~nm}$. Therefore, the former one photon transition can be directly converted into the latter two photon transition. Nevertheless, we do not observe any shortening of nanosecond decay times of $5 \mathrm{~d}-4 \mathrm{f}$ emission up to $1 \%$ of Pr doping level in YAP host (about 8 ns at RT [22]). In addition, only $\sim 25 \%$ shorter decay time is obtained for Pr-5\%(mPD,Ir) sample. Therefore, the possibility that cross-relaxation process involves the relaxed $5 \mathrm{~d}_{1}$ level of $\mathrm{Pr}^{3+}$ is rather unlikely, because the intensity ratio of the above mentioned d-f and f-f emissions changes from 1: 0.07 in Pr- $0.1 \%$ (mPD, Ir) to 1:0.5 in Pr-5\%(mPD,Ir) sample and absolute intensity of $5 \mathrm{~d}-4 \mathrm{f}$ 
TABLE I

Photoelectron Yield and Relative Scintillation EfFiciency of PR-Doped YAP SAMPles in Comparison With YAP:Ce StandaRd

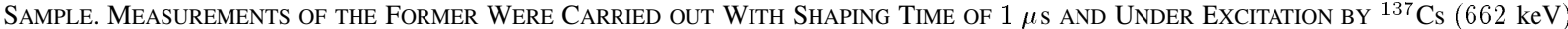
RADIOISOTOPE AND ${ }^{241} \mathrm{Am}(59.6 \mathrm{keV})$ FOR THE CZ-GROWN SAMPLES AND mPD GROWN SAMPLES, RESPECTIVELY. THE VALUES OF THE LATTER ARE DERIVED FROM THE INTEGRAL OF RADIOLUMINESCENCE SPECTRA, SEE FIGS. 1 AND 6.

\begin{tabular}{|c|c|c|c|c|c|c|}
\hline Sample & $\begin{array}{l}\text { Concentr. } \\
\text { Melt (\%) }\end{array}$ & $\begin{array}{c}\text { Growth } \\
\text { method } \\
\text { \&Crucible }\end{array}$ & $\begin{array}{c}\mathrm{N}_{\text {phels }} / \mathrm{MeV} \\
\text { (phels per } \\
\text { MeV) }\end{array}$ & $\begin{array}{c}\text { FWHM } \\
\text { (\%) at } \\
662 \mathrm{keV} \\
\end{array}$ & $\begin{array}{c}\mathrm{N}_{\text {phels }} \\
\% \text { of } \\
\text { standard }\end{array}$ & $\begin{array}{c}\mathrm{RL} \text { intens. } \\
\% \text { of } \\
\text { standard }\end{array}$ \\
\hline $\begin{array}{l}\text { YAP:Ce } \\
\text { Japan }\end{array}$ & $\mathrm{Ce}-0.05$ & $\mathrm{CZ}-\mathrm{Ir}$ & 1978 & 7.3 & 71 & 81 \\
\hline $\begin{array}{l}\text { YAP:Ce } \\
\text { Czech }\end{array}$ & Ce-0.5 & $\begin{array}{l}\mathrm{CZ}-\mathrm{Mo} \\
\text { standard }\end{array}$ & 2803 & 4.8 & 100 & 100 \\
\hline $\begin{array}{l}\text { YAP:Pr } \\
\text { Japan }\end{array}$ & $\operatorname{Pr}-0.05$ & $\mathrm{CZ}-\mathrm{Ir}$ & 2280 & 7.1 & 81 & 147 \\
\hline $\begin{array}{l}\text { YAP:Pr } \\
\text { Japan }\end{array}$ & $\operatorname{Pr}-1.0$ & $\mathrm{CZ}-\mathrm{Ir}$ & 1026 & 8.9 & 37 & 113 \\
\hline $\begin{array}{l}\text { YAP:Pr } \\
\text { Czech }\end{array}$ & $\operatorname{Pr}-0.5$ & $\mathrm{CZ}-\mathrm{Mo}$ & 975 & 10.3 & 35 & 130 \\
\hline $\begin{array}{l}\text { YAP:Pr } \\
\text { Czech }\end{array}$ & $\operatorname{Pr}-1.1$ & $\mathrm{CZ}-\mathrm{Mo}$ & 1127 & 9.1 & 40 & n.m. \\
\hline & & \multicolumn{2}{|c|}{ mPD - grown samples } & $\begin{array}{c}\text { FWHM } \\
(\%) \text { at } \\
59.6 \mathrm{keV}\end{array}$ & & \\
\hline $\begin{array}{l}\text { YAP:Pr } \\
\text { Japan }\end{array}$ & Pr-0.05 & $\mathrm{mPD}-\mathrm{Ir}$ & 1015 & 46.3 & 36 & 91 \\
\hline $\begin{array}{l}\text { YAP:Pr } \\
\text { Japan }\end{array}$ & $\operatorname{Pr}-0.1$ & mPD - Ir & 1203 & 54.9 & 43 & 96 \\
\hline $\begin{array}{l}\text { YAP:Pr } \\
\text { Japan }\end{array}$ & $\begin{array}{c}\text { Pr }-0.5 \\
\text { Hf 30ppm }\end{array}$ & mPD - Ir & 619 & 40.7 & 22 & 56 \\
\hline $\begin{array}{l}\text { YAP:Pr } \\
\text { Japan }\end{array}$ & $\begin{array}{c}\mathrm{Pr}-0.5 \\
\mathrm{Hf} 60 \mathrm{ppm}\end{array}$ & $\mathrm{mPD}-\mathrm{Ir}$ & 961 & 51.3 & 34 & n.m. \\
\hline $\begin{array}{l}\text { YAP:Pr } \\
\text { Japan }\end{array}$ & $\begin{array}{c}\text { Pr }-0.5 \\
\text { Hf120ppm }\end{array}$ & mPD - Ir & 1262 & $\sim 25$ & 45 & 102 \\
\hline $\begin{array}{l}\text { YAP:Pr } \\
\text { Japan }\end{array}$ & $\begin{array}{c}\mathrm{Pr}-0.5 \\
\mathrm{Hf} 300 \mathrm{ppm}\end{array}$ & $\mathrm{mPD}-\mathrm{Ir}$ & 1270 & $\sim 30$ & 45 & 64 \\
\hline $\begin{array}{l}\text { YAP:Pr } \\
\text { Japan }\end{array}$ & $\begin{array}{l}\mathrm{Pr}-0.5 \\
\mathrm{Mo}-0.05\end{array}$ & mPD - Ir & 492 & 35.1 & 18 & 45 \\
\hline
\end{tabular}

n.m. $=$ not measured

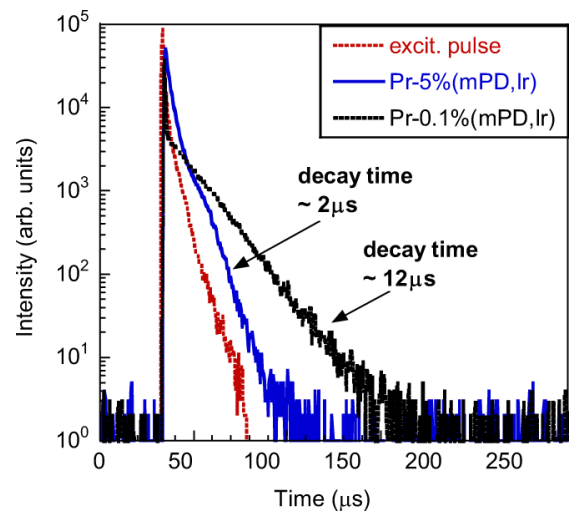

Fig. 2. RT photoluminescence decay of the $490 \mathrm{~nm}$ emission line, $\lambda_{\text {exc }}=$ $450 \mathrm{~nm}$.

emission decreases more than six times in the latter sample [22]. Moreover, a strong quenching of the $4 \mathrm{f}-4 \mathrm{f}$ emission lines is evidenced by decay times shortening from $12 \mu \mathrm{s}$ and $140 \mu \mathrm{s}(\operatorname{Pr}$ $0.1 \%$ ) to $2 \mu \mathrm{s}$ and $1-2 \mu \mathrm{s}$ (Pr5\%) in the $490 \mathrm{~nm}$ and $620 \mathrm{~nm}$ 4f $-4 \mathrm{f}$ emission lines, respectively, see Fig. 2 and [23].

The $\mathrm{f}-\mathrm{f}$ emission of $\mathrm{Pr}^{3+}$ in YAP is of interest mainly for lasing [24] or upconversion-related [25] purposes. However, it can also be of interest in the present case, since possible pairing or clustering of Pr ions in YAP host could be monitored by the

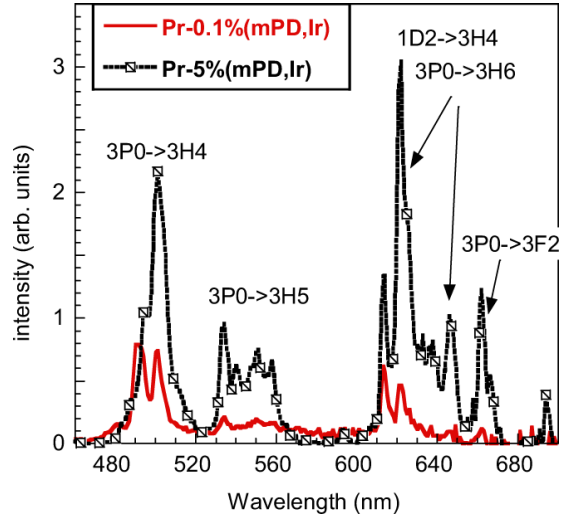

Fig. 3. RT $4 \mathrm{f}-4 \mathrm{f}$ photoluminescence spectra of Pr-doped YAP, $\lambda_{\text {exc }}=$ $450 \mathrm{~nm}$.

change of mutual intensities of the $\mathrm{f}-\mathrm{f}$ emission lines (e.g., in ranges from 490 to $510 \mathrm{~nm}$ and from 610 to $630 \mathrm{~nm}$ ). Mutual emission line intensities apparently depend on Pr concentration and may reflect changes in the symmetry of Pr site and its nearest neighbourhood, see Figs. 1 and 3.

Scintillation decay of the YAP:Pr samples (the set presented in Fig. 1) shows a typical two-exponential character, see Fig. 4. Since the slower component with the decay time of about several hundred ns can hardly be distinguished, its decay time and 


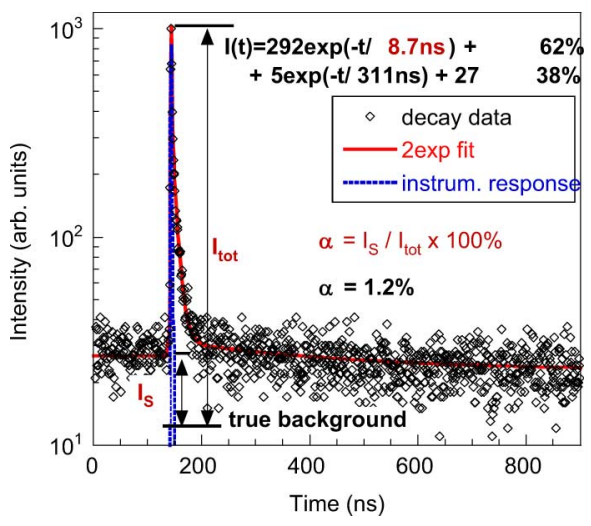

Fig. 4. RT scintillation decay of Pr-1\%(Cz,Ir) sample, spectrally unresolved, excited by $511 \mathrm{keV}$ photons of ${ }^{22} \mathrm{Na}$ radioisotope. Solid line is the convolution of instrumental response and function $\mathrm{I}(\mathrm{t})$ in the figure. Faster and slower component percentage fractions of total intensity are shown next to the $\mathrm{I}(\mathrm{t})$ formula. A construction of the coefficient alpha is sketched, see also [26]. True background level is given by the electronic noise of the photodetector.

calculated relative intensity of $38 \%$ are only indicative. Nevertheless, even much slower decay components do exist. Their decay time is comparable with the inverse repetition rate of the excitation pulse (10-20 kHz) as reflected by the increased level of the "background" before the signal rising edge (cf. Fig. 4). The fraction of intensity contained in slow components is expressed by the alpha coefficient, see [26] for details.

The dominant component has a decay time 8-9 ns, and releases about $40 \%$ of light intensity (calculated from the fit of the decay curve), the values consistent with the previous data [8]-[12]. Such fast decay promotes YAP:Pr to the category of the fastest scintillators based on $5 \mathrm{~d}-4 \mathrm{f}$ radiative transition in the UV-visible spectral range.

Preparation of a numerous set of the codoped samples of YAP:Pr was enabled by the flexible and fast $\mathrm{mPD}$ growth technique [27]. Some scintillation characteristics of the $\mathrm{Zr}$ and $\mathrm{Si}$ codoped YAP:Pr have been already published in our previous work [9], [28]. As mentioned in Section II the aim of codoping is to change the internal equilibrium of the YAP host defects due mainly to cation nonstoichiometry, presence of antisite defects and lack of oxygen anions. Their presence is determined by the technology, namely by the melt stoichiometry, growth atmosphere and post-growth treatments. The $\mathrm{Mg}^{2+}, \mathrm{Si}^{4+}, \mathrm{Zr}^{4+}$, $\mathrm{Hf}^{4+}, \mathrm{Mo}^{3+(4+)}$ and $\mathrm{Ga}^{3+}$ codopants were tested and their influence on the optical, luminescence and scintillation characteristics of YAP:Pr was studied.

In the absorption spectra of codoped crystals the parasitic (defect-related) absorption bands are frequently observed. Some examples are given in Fig. 5. In the as-grown $\mathrm{Pr}-0.1 \%$ $\mathrm{Mg}-0.01 \%$ (mPD,Ir) sample and in several other samples annealed in air at $1200^{\circ} \mathrm{C}$ for several hours a broad absorption band extending up to $600 \mathrm{~nm}$ was observed. It was reported that absorption bands around $2.1-2.3 \mathrm{eV}$ and $3.1-3.3 \mathrm{eV}$ can be related to the $\mathrm{O}^{-}$-based hole centers in YAP structure [29], [30]. At the same time the latter band can be also related to charge transfer transition from the valence band (oxygen ligands) towards $\mathrm{Pr}^{4+}$ center [31], [32]. $\mathrm{F}^{+}$center (one electron in oxygen vacancy) has been recently proved in YAP structure by EPR experiment [14] and can contribute to the observed absorption

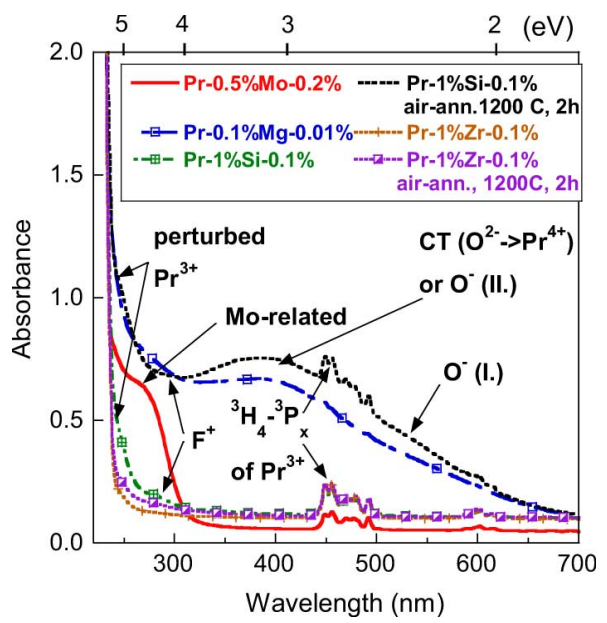

Fig. 5. Absorption spectra of selected codoped YAP:Pr crystals prepared by mPD growth technique. The position and tentative origin of the absorption bands are indicated.

around $4.3 \mathrm{eV}$ [33]. The smooth structureless absorption within 235-260 nm, adjacent to the edge of ${ }^{3} \mathrm{H}_{4}-5 \mathrm{~d}_{1}$ absorption transition of $\mathrm{Pr}^{3+}$ at $5.3 \mathrm{eV}(234 \mathrm{~nm})$, is most probably related to the same absorption transition of slightly perturbed $\mathrm{Pr}^{3+}$ ions which is very well known issue e.g., in $\mathrm{CeF}_{3}$ single crystals [3]. Only in the Mo-codoped samples an absorption band around $4.6 \mathrm{eV}$ appears. Its amplitude correlates with the Mo concentration in the melt. It can be assigned to the charge transfer transition between the valence band and $\mathrm{Mo}^{4+}$ and/or between $\mathrm{Mo}^{3+}$ and the conduction band [34]. Apparently, most of these absorption bands overlap with the $5 \mathrm{~d}_{1}-4 \mathrm{f}$ emission of $\mathrm{Pr}^{3+}$ so that their presence in bulk crystals must be minimized. A specific problem of the YAP structure consists in negative effect of cationic nonstoichiometry as it always results in the presence of triply charged cation vacancies which can stabilize various $\mathrm{O}^{-}$-based centers nearby. Such centers are indeed observed with the help of EPR measurements [14], [22].

Radioluminescence spectra of practically all the codoped samples show clear enhancement of the $4 \mathrm{f}-4 \mathrm{f}$ emission lines (relative to $5 \mathrm{~d}_{1}-4 \mathrm{f}$ emission) with respect to samples without codoping. The reduction of absolute intensity of the $5 \mathrm{~d}_{1}-4 \mathrm{f}$ band occurs as well, see Fig. 6 and Table I. Comparing crystals with different codopants, the most promising result was obtained for the Hf-codoped samples with Hf concentration around $120-160 \mathrm{ppm}$. Their spectral shape and RL intensity are comparable or slightly superior to those of the Pr-only doped YAP grown by the mPD method, see Fig. 6. It is interesting to note that similar concentration of trivalent dopants in $\mathrm{PbWO}_{4}$ was found to provide maximum optimization of scintillation performance mainly due to the suppression of oxygen vacancies and unknown deep hole traps, see [3], [13] for the review.

The scintillation decay of codoped samples shows the similar shape and decay time values as those displayed in Fig. 4. In the case of $\mathrm{Zr}$ and $\mathrm{Hf}$ codoping the fast component becomes somewhat more intense (containing about $60-70 \%$ of total intensity calculated from the decay curve fit).

Photoelectron yield, energy resolution and proportionality of the $\mathrm{N}_{\text {phels }}$ yield were measured for an extended set of samples, see Table I and [9]. In the upper and bottom part of Table I 


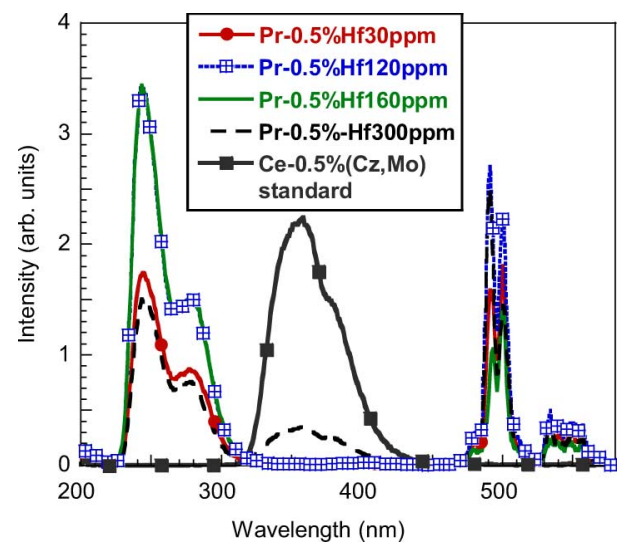

Fig. 6. Radioluminescence of the Hf-codoped YAP:Pr compared to the Ce-0. $5 \%(\mathrm{Cz}, \mathrm{Mo})$ standard sample. Excitation by X-ray tube, $40 \mathrm{kV}$, RT. Spectra are mutually comparable in an absolute scale.

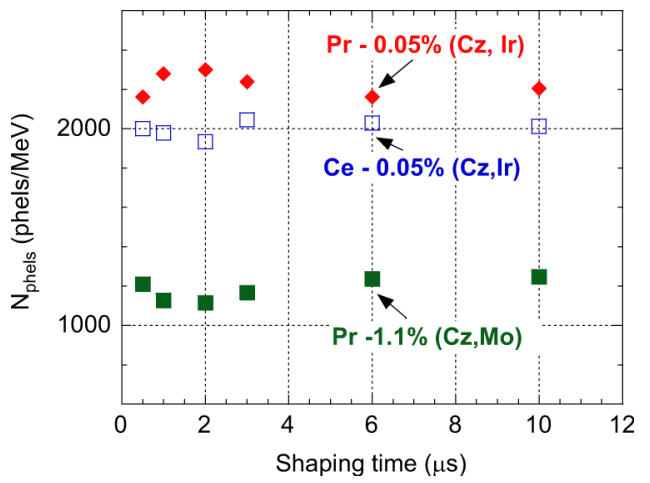

Fig. 7. $\mathrm{N}_{\text {phels }}$ per $1 \mathrm{MeV}$ photoelectron yield time development of the selected Ce- and Pr-doped YAP. Error of the measured values is $\pm 5 \%$.

the Cz-grown and mPD-grown samples are grouped, respectively. In the case of $\mathrm{N}_{\text {phels }}$ and scintillation efficiency values the mPD-grown samples are clearly performing worse. Dependence of $\mathrm{N}_{\text {phels }}$ yield on the shaping time for selected Ce- or $\mathrm{Pr}$-doped $\mathrm{Cz}$ grown crystals is presented in Fig. 7. As evident, there is no observable difference among these crystals. Rather flat observed time dependence of $\mathrm{N}_{\text {phels }}$ is consistent with the shape of scintillation decay in Fig. 4 where only barely visible slower decay component is present. In case of the energy resolution in $\mathrm{Cz}$ grown crystal set the best $\mathrm{Ce}-0.5 \%$ standard sample $(4.8 \%$ at $662 \mathrm{keV})$ is outperforming the best $\mathrm{Pr}-0.05 \%$ doped crystal $(7.1 \%$ at $662 \mathrm{keV})$ which might be influenced by somewhat lower $\mathrm{N}_{\text {phels }}$ value of the latter.

The proportionality of the $\mathrm{N}_{\text {phels }}$ yield is shown in Fig. 8 for selected Ce- and Pr-doped YAP samples. In this case the proportionality of the Ce- $0.5 \%$ standard sample is worse than the best $\mathrm{Pr}-0.05 \%$ doped crystal. The proportionality of other $\mathrm{Cz}$ grown YAP:Ce from Mo crucible is presented in [17] and its proportionality is found between 90 and $102 \%$ in the same range of energies.

The last two columns of Table I report the relative comparison of scintillation efficiency and photoelectron yield of all the samples with those of the $\mathrm{Ce}-0.5 \%(\mathrm{Cz}, \mathrm{Mo})$ standard sample. One can notice an apparent inconsistency in the high scintillation efficiency (proportional to the integrated $5 \mathrm{~d}-4 \mathrm{f}$ radioluminescence spectra measured in an absolute scale as in Figs. 1 and 6)

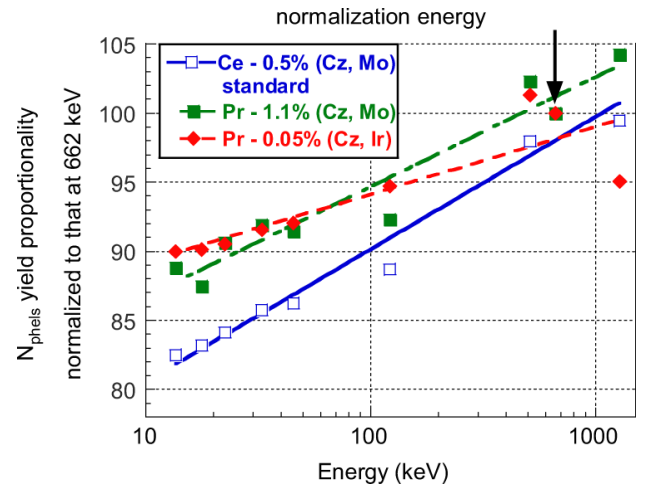

Fig. 8. The non-proportionality of the $\mathrm{N}_{\text {phels }}(\mathrm{E})$ photoelectron yield of Ce-doped crystals (dimensions $\varnothing 10 \times 10 \mathrm{~mm}^{3}$ ). Data normalized at $662 \mathrm{keV}$. Error of the measured values is $\pm 5 \%$.

and much lower photoelectron yield for all the Pr-doped YAPs. This can be explained assuming that in the Pr-doped samples there is an enhanced delayed radiative recombination at $\operatorname{Pr}^{3+}$ centers due to unidentified trapping processes in the transport stage of the scintillation mechanism. Such processes must occur in the time scale of several tens of microseconds or longer as suggested by the above mentioned increased background signal in the scintillation decay measurement monitored by the alpha coefficient [26].

The presence of shallow trapping states in a scintillator can be monitored by TSL measurements below RT. Moreover, the correlation between TSL and EPR measurements can shed light on the nature of the involved trapping states, but only if they are paramagnetic. Unfortunately EPR cannot be used to monitor the presence of $\mathrm{Pr}^{3+}$ since it is not paramagnetic; moreover, to our knowledge there are also no EPR data in the literature for $\mathrm{Pr}^{4+}$ which has the same electronic configuration as the $\mathrm{Ce}^{3+}$ paramagnetic center. Low temperature wavelength-resolved TSL measurements were performed on some of the here reported samples [35]. In Fig. 9 we show typical glow curves of undoped, Ce-doped and Pr-doped YAP. In undoped YAP the glow peaks within $150-250 \mathrm{~K}$ were assigned to defect-stabilized O- centers [14], [34], [35].1 The proposed recombination process involves the thermal release of holes from specific trap and their radiative recombination with electrons stored in oxygen vacancies (giving rise to TSL glow peaks) and/or their localization in deeper traps. In the case of Ce or Pr-doped samples the glow curve changes noticeably. It is the consequence of the supposed hole trap character of both $\mathrm{Ce}^{3+}$ and $\mathrm{Pr}^{3+}$ ions which compete with intrinsic $\mathrm{O}^{-}$-based traps in the hole capture. However, unlike in aluminum garnets where TSL glow curves of Ce and Pr-doped samples are similar [36], they manifest considerable differences in YAP host. As discussed in detail in Ref. 35, a kind of complex defect is created around $\mathrm{Ce}^{3+}$ and $\mathrm{Pr}^{3+}$ dopant ion, in which electron and hole traps are spatially correlated as also evidenced by an additional athermal tunneling recombination process (flat structureless TSL signal occurring at temperatures lower than the principal TSL peak). TSL spectra display only the well-known bands of $\mathrm{Ce}^{3+}$ or $\mathrm{Pr}^{3+}$ [35] demonstrating that the final radiative recombination occurs at the doped rare earth ion. However, the composition and/or 


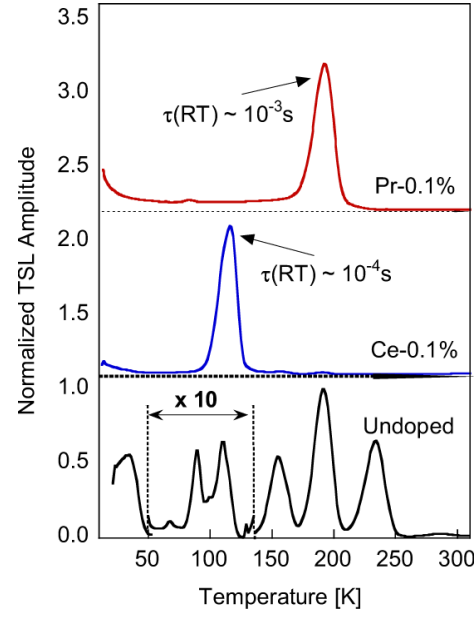

Fig. 9. TSL glow curves after $\mathrm{x}$-irradiation at $10 \mathrm{~K}$ of undoped, Ce-doped and Pr-doped YAP. See also [29].

configuration of such a complex defect does not seem to be the same for both $\mathrm{Ce}^{3+}$ and $\mathrm{Pr}^{3+}$ dopants. Nevertheless, an important conclusion can be drawn from Fig. 9: as the dominant glow curve peak occurs at a much higher temperature in the case of $\mathrm{Pr}$ doping, the lifetime of the filled trap at RT is about one order of magnitude longer with respect to that in Ce-doped YAP (compare the values reported in Fig. 9). This can be one of the reasons why the mentioned delayed radiative recombination processes in YAP:Pr are more pronounced and the photoelectron yield is lower with respect to that of YAP:Ce, despite the higher scintillation efficiency evaluated from YAP:Pr radioluminescence spectra.

\section{CONCLUSION}

The scintillation performance of a numerous set of Pr-doped YAP single crystals prepared by three different technologies was evaluated by radioluminescence, photoelectron yield and scintillation decay measurements. The intrinsic scintillation efficiency of the best Czochralski grown YAP:Pr reached about $150 \%$ of that of YAP:Ce standard sample. However, its photoelectron yield is only about $80 \%$ of that of YAP:Ce standard suggesting the presence of enhanced delayed radiative recombination processes in YAP:Pr. The discrepancy between scintillation efficiency and photoelectron yield is systematically observed in all the Pr-doped samples. The mPD grown samples show on average an inferior quality with respect to the Czochralski grown ones. Thermoluminescence measurements in the $10-350 \mathrm{~K}$ temperature range manifest that in YAP:Pr the dominant glow curve peak occurs at a noticeably higher temperature $(192 \mathrm{~K})$ with respect to that found in YAP:Ce $(115 \mathrm{~K})$. The calculated RT lifetime of the $192 \mathrm{~K}$ peak-related trap in YAP:Pr is about one order of magnitude longer $(1 \mathrm{~ms})$ than that related to the $115 \mathrm{~K}$ peak in YAP:Ce. It can be one of the reasons of the decrease of photoelectron yield in YAP:Pr due to an enhanced delay in the transport stage of scintillation mechanism. However, since the existence of the mentioned delayed radiative recombination processes in YAP:Pr is due to a defect instead of being an intrinsic property of this material, the possibilities to further optimize the scintillation performance of YAP:Pr remain open. As its intrinsic scintillation efficiency even exceeds that of YAP:Ce, also its photoelectron yield could become superior if the degrading defects and related traps are suppressed.

So far performed codoping of YAP:Pr prepared by micropulling down method did not manifest an evident improvement of the scintillation figure-of-merit even if the results achieved with the Hf codoping are encouraging. The codoping effect must both consistently and quantitatively balance possible material (non)stoichiometry and potential lack of oxygen in anion sublattice which could be the crucial issue in further technology optimization and improvement of YAP:Pr scintillation performance.

\section{REFERENCES}

[1] M. J. Weber, "Optical spectra of $\mathrm{Ce}^{3+}$ and $\mathrm{Ce}^{3+}$-sensitized fluorescence in $\mathrm{YAlO}_{3}$," J. Appl. Phys., vol. 44, pp. 3205-3208, 1973.

[2] E. Autrata, P. Schauer, J. Kvapil, and J. Kvapil, "A single crystal of $\mathrm{YAlO}_{3}: \mathrm{Ce}^{3+}$ as a fast scintillator SEM," Scanning, vol. 5, pp. 91-96, 1983.

[3] M. Nikl, "Wide band gap scintillation materials. Progress in the technology and material understanding," Phys. stat. sol. (a), vol. 178, pp. 595-620, 2000.

[4] P. A. Rodnyi, A. N. Mishin, and A. S. Potapov, "Luminescence of trivalent praseodymium in oxides and fluorides," Opt. Spectr., vol. 93, pp. 714-721, 2002.

[5] C. W. E. van Eijk, P. Dorenbos, and R. Visser, " $\mathrm{Nd}^{3+}$ and $\mathrm{Pr}^{3+}$ doped inorganic scintillators," IEEE Trans. Nucl. Sci., vol. 41, pp. 738-741, 1994.

[6] J. Pejchal, M. Nikl, E. Mihóková, J. A. Mareš, A. Yoshikawa, H. Ogino, K. M. Schillemat, A. Krasnikov, A. Vedda, K. Nejezchleb, and V. Múčka, "Pr ${ }^{3+}$-doped complex oxide single crystal scintillators," $J$. Phys. D: Appl. Phys., vol. 42, p. 055117, 2009.

[7] M. Nikl, H. Ogino, A. Krasnikov, A. Beitlerova, A. Yoshikawa, and T. Fukuda, "Photo-and radioluminescence of Pr-doped $\mathrm{Lu}_{3} \mathrm{Al}_{5} \mathrm{O}_{12}$ single crystal," Phys. Stat. sol. (a), vol. 202, pp. R4-R6, 2005.

[8] E. G. Gumanskaja, M. V. Korzhik, A. S. Smirnova, B. V. Pavlenko, and A. A. Fedorov, "Mezhkonfiguracionaja luminescencija $\operatorname{Pr}^{3+} \mathrm{v}$ monokristalach $\mathrm{Y}_{3} \mathrm{Al}_{5} \mathrm{O}_{12}$ i $\mathrm{YALO}_{3}$," Opt. Spectrosc., vol. 72, pp. 155-159, 1992.

[9] M. Zhuravleva, A. Novoselov, E. Mihokova, J. A. Mares, A. Vedda, M. Nikl, and A. Yoshikawa, "Crystal growth and scintillating properties of $\mathrm{Zr} / \mathrm{Si}$-codoped $\mathrm{YAlO}_{3}$ : $\mathrm{Pr}^{3+}$," IEEE Trans. Nucl. Sci., vol. 55, pp. 1476-1479, 2008.

[10] V. G. Barishevski, G. Y. Drobyshev, A. A. Fyodorov, M. V. Korzhik, V. I. Moroz, V. B. Pavlenko, C. W. E. van Eijk, P. Dorenbos, S. A. Smirnova, O. A. Egorycheva, O. G. Savinova, and V. A. Kachanov, "Rare-earth aluminium perovskite scintillators," in Proc. Of CRYSTAL2000 Int. Workshop 1992 Chamonix, France, pp. 195-199.

[11] C. Dujardin, C. Pedrini, J. C. Gacon, A. G. Petrosyan, A. N. Belski, and A. N. Vasil'ev, "Luminescence properties and scintillation mechanism of cerium and preseodymium-doped lutetium orthoaluminate," $J$. Phys. Cond. Matter, vol. 9, pp. 5229-5243, 1997.

[12] W. Drozdowski, A. J. Wojtowicz, D. Wisniewski, T. Lukasiewicz, and J. Kisielewski, "Scintillation properties of Pr-activated $\mathrm{LuAlO}_{3}$," Opt. Mater., vol. 28, pp. 102-105, 2006.

[13] M. Nikl, A. Vedda, and V. V. Laguta, "Energy transfer and storage processes in scintillators: The role and nature of defects," Radiation Measurements, vol. 42, pp. 509-514, 2007.

[14] V. V. Laguta, M. Nikl, A. Vedda, E. Mihokova, J. Rosa, and K. Blazek, "The hole and electron traps in the $\mathrm{YAlO}_{3}$ single crystal scintillator," Phys. Rev. B, vol. 80, p. 045114, 2009.

[15] M. Nikl, E. Mihokova, J. Pejchal, A. Vedda, M. Fasoli, I. Fontana, V. V. Laguta, V. Babin, K. Nejezchleb, A. Yoshikawa, H. Ogino, and G. Ren, "Scintillator materials - achievements, opportunities, and puzzles,” IEEE Trans. Nucl. Sci., vol. 55, pp. 1035-1041, 2008.

[16] L. M. Bollinger and G. E. Thomas, "Measurement of the time dependence of scintillation intensity by a delayed-coincidence method," Rev. Sci. Instrum., vol. 32, pp. 1044-1050, 1961. 
[17] J. A. Mares, A. Beitlerova, M. Nikl, N. Solovieva, C. D'Ambrosio, K. Blazek, P. Maly, K. Nejezchleb, and F. de Notaristefani, "Scintillation response of Ce-doped or intrinsic scintillating crystals in the range up to 1 Mev," Radiation Measurements, vol. 38, pp. 353-357, 2004.

[18] C. D'Ambrosio and J. A. Mares, "Hybrid photomultipliers- their properties and application in scintillation studies," Opt. Mat., vol. 30, pp. $22-25,2007$.

[19] J. B. Shim, A. Yoshikawa, M. Nikl, N. Solovieva, J. Pejchal, D. H. Yoon, and T. Fukuda, "Growth and characterization of $\mathrm{Yb}^{3+}$-doped $\mathrm{YAlO}_{3}$ fiber single crystals grown by the modified micro-pulling-down method," J. Cryst. Growth, vol. 256, pp. 298-304, 2003.

[20] M. Nikl, N. Solovieva, J. Pejchal, J. B. Shim, A. Yoshikawa, T. Fukuda, A. Vedda, M. Martini, and D. H. Yoon, "Very fast $\mathrm{Yb}_{x} \mathrm{Y}_{1-x} \mathrm{AlO}_{3}$ single crystal scintillators," Appl. Phys. Letters, vol. 84, pp. 882-884, 2004.

[21] B. M. Eppelbaum, G. Schierning, and A. Winnacker, J. Cryst. Growth, vol. 275, pp. e867-870, 2005.

[22] M. Zhuravleva, A. Novoselov, A. Yoshikawa, J. Pejchal, M. Nikl, and T. Fukuda, "Crystal growth and scintillation properties of Pr-doped $\mathrm{YAlO}_{3}$, , Optical Materials, vol. 30, pp. 171-173, 2007.

[23] R. Balda, J. Fernandez, I. Saez de Ocariz, M. Voda, and A. J. Garcýa, "Laser spectroscopy of $\mathrm{Pr}^{3+}$ ions in $\mathrm{LiKY}_{1-x} \mathrm{Pr}_{x} \mathrm{~F}_{5}$ single crystals," Phys. Rev. B, vol. 59, pp. 9972-9980, 1999.

[24] H. Jelinkova, M. Fibrich, M. Cech, P. Hirsl, K. Nejezchleb, and V. Skoda, "Electro-optically Q-switched Pr:YAP laser generating at 747 nm," Laser Phys. Lett., pp. 1-4, 2009.

[25] G. Ozen, O. Forte, B. Di Bartolo, and J. M. Collins, "Dynamics of the excitation and upconversion processes in $\mathrm{YAlO}_{3}$ : Pr single crystals," $J$. Lumin., vol. 125, pp. 223-229, 2007.

[26] M. Nikl, "Energy transfer phenomena in the luminescence of wide band-gap scintillators," Phys. stat. sol. (a), vol. 202, pp. 201-206, 2005.

[27] A. Yoshikawa, M. Nikl, G. Boulon, and T. Fukuda, "Challenge and study for developing of novel single crystalline optical materials using micro-pulling-down method," Optical Materials, vol. 30, pp. 6-10, 2007.
[28] M. Zhuravleva, A. Novoselov, E. Mihokova, J. A. Mares, A. Vedda, M. Nikl, and A. Yoshikawa, "Crystal growth and scintillating properties of (Pr,Si)-doped $\mathrm{YAlO}_{3}$, , Cryst. Res. Technol., vol. 42, pp. 1324-1328, 2007.

[29] O. F. Schirmer, K. W. Blazey, and W. Berlinger, "ESR amd optical absorption of bound-small polarons in $\mathrm{YAlO}_{3}$, Phys. Rev. B, vol. 11, pp. 4201-4211, 1975.

[30] M. Nikl, E. Mihokova, V. Laguta, J. Pejchal, S. Baccaro, and A. Vedda, L. Juha, R. H. Sobierajski, and H. Wabnitz, Eds., "Radiation damage processes in complex-oxide scintillators. European symposium on optics and optoelectronics: Damage to VUV, EUV, and X-ray optics," in Proc. of SPIE, 2007, vol. 6586, p. 65860E.

[31] D. Pawlak, Z. Frukacz, Z. Mierczyk, A. Suchocki, and J. Zachara, "Spectroscopic and crystallographic studies of YAG:Pr single crystals," J.All.Comp., vol. 275-277, pp. 361-364, 1998.

[32] D. A. Pawlak, T. Lukasiewicz, M. Carpenter, M. Malinowski, R. Diduszko, and J. Kisielewski, "Czochralski crystal growth, microstructureand spectroscopic properties of $\mathrm{PrAlO}_{3}$ perovskite," $J$. Cryst.Growth, vol. 282, pp. 260-269, 2005.

[33] V. Yu, A. S. Zorenko, I. Voloshinovskii, and V. Konstankevych, "Luminescence of $\mathrm{F}^{+}$and $\mathrm{F}$ centers in $\mathrm{YAlO}_{3}$," Opt. Spectr., vol. 96, pp. 532-537, 2000.

[34] M. Nikl, V. V. Laguta, and A. Vedda, "Complex oxide scintillators: Material defects and scintillation performance," Phys. Stat. sol. (b), vol. 245, pp. 1701-1722, 2008

[35] A. Vedda, M. Fasoli, M. Nikl, V. V. Laguta, E. Mihokova, J. Pejchal, A. Yoshikawa, and M. Zhuravleva, "Trap-centre recombination processes by rare earth activators in $\mathrm{YAlO}_{3}$ single crystal host," Phys. Rev. B, vol. 80, p. 045113, 2009.

[36] M. Nikl, J. Pejchal, E. Mihokova, J. A. Mares, H. Ogino, A. Yoshikawa, T. Fukuda, A. Vedda, and C. D'Ambrosio, "Antisite defect-free $\mathrm{Lu}_{3}\left(\mathrm{Ga}_{x} \mathrm{Al}_{1-x}\right)_{5} \mathrm{O}_{12}$ :Pr scintillator," Appl. Phys. Letters, vol. $88,2006,141916$. 